OPTIMISING MANAGEMENT AND IMPROVING OUTCOMES IN CHRONIC OBSTRUCTIVE PULMONARY DISEASE BY INTEGRATING CARE

doi:10.1136/thoraxjnl-2011-201054c.97

\section{P98 IMPROVING DIAGNOSIS AND MANAGEMENT OF COPD: LEARNING FROM A PROGRAMME OF NATIONAL IMPROVEMENT PROJECTS}

doi:10.1136/thoraxjnl-2011-201054c.98

${ }^{1}$ E J Cetti, ${ }^{2}$ E Vijaykumar. ${ }^{1}$ East Surrey Hospital, Redhill, UK; ${ }^{2}$ ESyDoc GP Consortium, Redhill, UK

Introduction/Objectives Previous integrated care models for COPD have reduced length of stay slightly ${ }^{1}$ or improved patient quality of life. In this study a pathfinder consortium of 20 practices and the local acute hospital, implemented a collaborative project with a partner from the pharmaceutical industry to improve COPD outcomes. The aims were to reduce hospital admissions, re-admissions and length of stay by integrating care via a patient focussed pathway.

Methods A care pathway was developed, involving patients, that crossed primary and secondary care boundaries and led to improved access to community respiratory services. In each practice patients with COPD were stratified by risk and reviewed by trained nurses, in a structured format. Practice Nurse educational needs were assessed and a mentorship programme put in place. A Consultant Respiratory Physician from the local hospital visited the practices to discuss COPD management and the care pathway, and now runs on-going education and support. National medicine management guidelines were adhered to and reinforced with all healthcare professionals. The links between primary care and the community respiratory team were enhanced and clear referral guidelines were disseminated. The local patient support group (Breathe Easy) was re-launched.

Results Patients were satisfied with the structured nurse-led COPD reviews, 463/487 said they were "very satisfied", and 433/487 said they were "totally aware" of their self-management plan. There was a $21 \%$ reduction in COPD hospital bed days and the average length of stay fell from 6.8 days to 5.0 days. At the end of 2010 the 30 day re-admission rate had fallen below the Strategic Health Authority average. Over the 12 months of the project the 90 day re-admission rate fell from $43 \%$ to $31 \%$, a $12 \%$ reduction not shown previously elsewhere ${ }^{1}$ - see Abstract P97 figure 1.

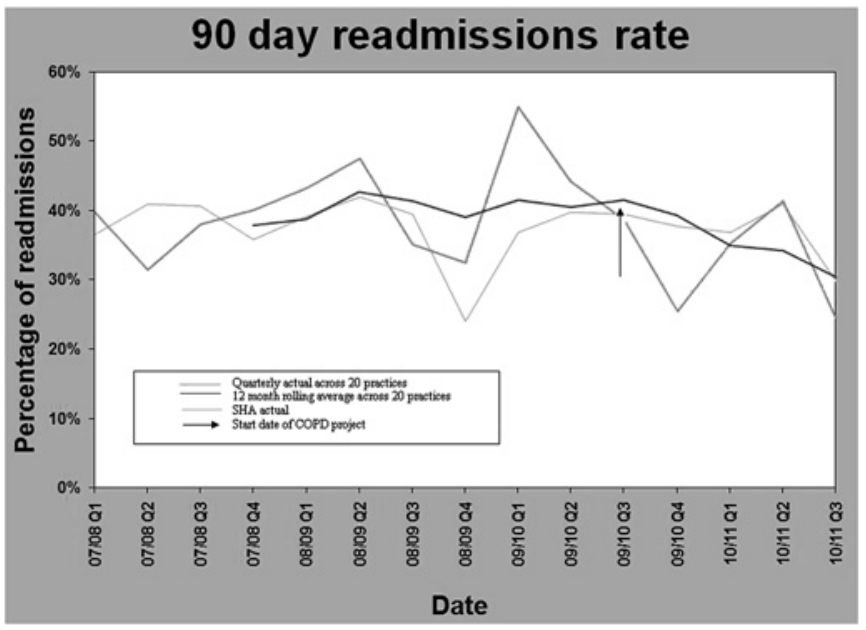

Abstract P97 Figure 1

Conclusions By engaging with all aspects of COPD care, an integrated multidisciplinary team improved service delivery and patient care, reducing COPD hospital bed days and re-admission rates. If the current proposed NHS reforms offer an opportunity for better integrated healthcare then they may deliver improved outcomes.

\section{REFERENCE}

1. NHS Quality Observatory.
C Blackaby, Z Lord, C Thompson, A Porter, P Duncan, 0 Okosi, H Wall. NHS Improvement, Leicester, UK

Introduction Chronic Obstructive Pulmonary Disease (COPD) represents an increasing burden for the NHS. National data indicate significant variation in the quality and consistency of diagnosis and management of COPD, with low recorded prevalence and increasing admissions. Early, accurate diagnosis and proactive management can modify disease progression to improve quality of life and use of health care resources. This national improvement programme aims to reduce variation and optimise diagnosis, treatment and use of healthcare resources through implementation of chronic disease management approaches

Methods 16 project sites from primary and secondary care adopted a systematic approach using improvement methodology to analyse existing patient pathways for COPD diagnosis and management, test changes and evaluate impact. Primary care and acute admission data, process mapping and patient feedback was analysed to identify risk, duplication and omissions in care and to evaluate the impact of changes implemented.

Results Great inaccuracy was identified in practice registers for COPD and asthma. Projects demonstrated that up to $69 \%$ of COPD patients also had a recorded diagnosis of asthma; up to $15 \%$ had no spirometry recorded and up to $40 \%$ had incomplete results. $21-43 \%$ of patients had an $\mathrm{FEV}_{1} / \mathrm{FVC}$ ratio $>0.7$, which is not indicative of COPD. Systematic process improvement of inpatient and community pathways in one project delivered savings of $£ 170 \mathrm{k}$ while medicines management reduced one practice's respiratory prescribing costs by $£ 1.5 \mathrm{k}$ per month. Consistent coding and use of self management support increased early identification of exacerbation and reduced the rate of COPD exacerbations resulting in admission from $8 \%$ to $5 \%$ in another project.

Conclusion Systematic improvement approaches can help reduce variation, improve quality, reduce admissions, and contain costs without significant impact on resources. Detailed practice-level information can demonstrate significant scope to improve the quality of diagnosis and reliability of appropriate treatment. Consistent coding in primary care is essential for proactive management of patients. Accurate diagnosis and regular review to optimise medicines use can deliver overall cost reductions. Further work is needed to demonstrate how teams can best use existing resources to deliver proactive management of COPD.

\section{P99 30-DAY COPD READMISSIONS RELATE TO DISEASE SEVERITY AND DEMOGRAPHIC FACTORS RATHER THAN SIMPLY ORGANISATION AND DELIVERY OF HOSPITAL CARE}

doi:10.1136/thoraxjnl-2011-201054c.99

J Hare, E Thompson, K Bodger, P Walker, M Pearson. Aintree Health Outcomes Partnership, University of Liverpool, Liverpool, UK

30-Day readmission rates after hospitalisation have risen and the UK Department of Health plans to introduce financial penalties for early readmissions. The surgical argument is reasonably clear but for chronic conditions such as COPD the presumption that early readmission is either avoidable or due to poor hospital care is far from clear. We used Hospital Episode Statistics data to examine the characteristics of patients readmitted within 30 days of an index COPD hospitalisation during 2006-2007. Mortality data were obtained from ONS death registration. There were 124834 COPD admissions in 2006-2007 with 78693 different patients discharged 
from hospital and we followed the first admission of each. 28533 (36\%) patients were readmitted within 1-year and 8439 (11\%) within 30 days. Patients readmitted within 30 days were more likely to be male (53\% vs $50 \%$; $<<0.001$ ), older (72.7 vs 71.6 yrs; $\mathrm{p}<0.001$ ), live in a more deprived area ( $55 \%$ vs $51 \%$ highest quintile; $p<0.001$ ) and much more likely to die (30-day mortality $11 \%$ vs $3 \%$; $<<0.001$; and 1 -year mortality $40 \%$ vs $20 \%$; $<0.001$ ). For the index admission, patients readmitted within 30 days were more likely to have been admitted via A\&E rather than via their GP (74\% vs $70 \%$; $\mathrm{p}<0.001)$, more likely to have had a LRTI ( $88 \%$ vs $83 \%$; $<<0.001)$, had a longer median length of stay ( 6 vs 5 days; $p<0.001$ ) and were more likely to have been discharged from the care of a chest physician (44\% vs $40 \%$; $p<0.001$ ). Hospitals with the lowest inpatient mortality during the first admission had the highest 30-day readmission rate $(r=0.22 ; p<0.01)$. Early readmission would appear to be driven by demographics, severity of the index admission and disease severity which cannot be influenced by care delivery or organisation of care. It's possible that better care-keeping more patients alive during their index admission-may lead to those hospitals being penalised for a higher 30-day readmission rate.

\section{P100 DESIGNING AND IMPLEMENTING A COPD DISCHARGE CARE BUNDLE}

doi:10.1136/thoraxjnl-2011-201054c.100

${ }^{1} \mathrm{~N}$ S Hopkinson, ${ }^{2} \mathrm{C}$ Englebretsen, ${ }^{1} \mathrm{~N}$ Cooley, ${ }^{3} \mathrm{~K}$ Kennie, ${ }^{1} \mathrm{M}$ Lim, ${ }^{2} \mathrm{~T}$ Woodcock, ${ }^{2} \mathrm{~A}$ Laverty, ${ }^{2} \mathrm{~S}$ Wilson, ${ }^{4} \mathrm{~S}$ L Elkin, ${ }^{2} \mathrm{C}$ Caneja, ${ }^{3} \mathrm{C}$ Falzon, ${ }^{2} \mathrm{H}$ Burgess, ${ }^{2} \mathrm{D}$ Bell, ${ }^{2} \mathrm{D}$ Lai. ${ }^{1}$ NIHR Respiratory Biomedical Research Unit of Royal Brompton and Harefield NHS Trust and Imperial College, London, UK; ${ }^{2}$ North West London Collaboration for Leadership in Applied Health Research and Care, Chelsea and Westminster NHS Foundation Trust, London, UK; ${ }^{3}$ Central London Community Healthcare, London, UK; ${ }^{4}$ Imperial Healthcare NHS Trust, London, UK

Problem Acute exacerbations of COPD are a major cause of hospital admission and readmission. National surveys have revealed significant differences in patient outcomes which are likely to be due to variations in care.

Design A care bundle, comprising a short list of evidence-based practices to be implemented prior to discharge for all patients admitted with this condition was developed, based on a review of National Guidelines and other relevant literature, expert opinion and patient consultation. Implementation was then piloted using action research methodologies in an urban acute hospital.

Key measures for improvement Improvements in process measures based around the items in the care bundle were the provision of smoking cessation advice, referral for pulmonary rehabilitation, and review of inhaler technique. A $72 \mathrm{~h}$ post-discharge phone call was evaluated. Data from the first year of the bundle were compared with the year prior to its initiation including 30-day readmission rate.

Strategies for change The care bundle was launched with events to ensure staff awareness. Weekly meetings were held with review of bundle compliance so that areas for attention could be addressed promptly. A plan, do, study, act (PDSA) cycle approach was used. Examples included ongoing support to develop ward nurses' knowledge of correct inhaler use, nurses attending pulmonary rehabilitation sessions and the use of a "safe discharge" checklist.

Effects of change Referral to pulmonary rehabilitation increased by $158 \%$ and compliance with assessment for smoking cessation was $100 \%$. Nurse confidence in inhaler technique improved. Roughly $10 \%$ of post discharge phone calls identified a cause for concern. The 30 -day readmission rate was $10.8 \%$ for patients where the bundle was used $(n=94)$ compared to $16.4 \%$ where it was not $(n=365)$ (95\% CI for difference $-2.1 \%$ to $13.2 \%$ ). The bundle has been accepted onto the list of CQUIN measures by London SHA and is being rolled out to further sites.
Lessons learnt Actively involving all staff is vital to ensure that the changes introduced are understood and the process followed. Implementation of a care bundle has the potential to produce a dramatic improvement in compliance with optimum health care measures.

\section{P101 A SURVEY OF EMERGENCY OXYGEN GUIDELINE IMPLEMENTATION AMONG ALL 15 UK AMBULANCE SERVICES IN EARLY 2011}

doi:10.1136/thoraxjnl-2011-201054c.101

${ }^{1} \mathrm{~F}$ P Moore, ${ }^{2} \mathrm{~A}$ G Davison, ${ }^{1} \mathrm{~J}$ Smith, ${ }^{3} \mathrm{~B}$ R O'Driscoll. 'London Ambulance Service, London, UK; ${ }^{2}$ Southend University Hospital, Southend, UK; ${ }^{3}$ Salford Royal University Hospital, Salford, UK

The British Thoracic Society (BTS), together with 21 other societies published a UK guideline for emergency oxygen use in 2008. This guideline was endorsed by the Joint Royal Colleges Ambulance Liaison Committee (JRCALC) who produced new oxygen guidance for ambulance crews in April 2009. We have conducted a survey of implementation of this guidance among UK Ambulance Services in early 2011. A questionnaire was sent to the Medical Directors of all 15 UK Ambulance Services and all, or their nominated deputies responded. Eleven of fifteen respondents reported full implementation of the 2009 JRCALC oxygen guidance throughout their service and ten of these reported that all relevant staff are trained in this area of practice. However, an informal survey of about 100 front line ambulance crews in one of these areas found that none were aware of the 2009 JRCALC document so the above figures may be aspirational and not yet achieved at operational level. Four services reported that they have completed audits of guideline implementation and a further three services are planning audits. All 15 services reported that $81 \%-100 \%$ of response vehicles were equipped with oximeters and the availability of oxygen masks was as follows: 15/15 reservoir masks; 12/15 simple face masks, 12/15 $28 \%$ Venturi masks, $6 / 15$ 24\% Venturi masks, 10/15 nasal cannulae. However, the informal survey of front-line staff from one ambulance service showed that Venturi masks were not actually available at operational level although the Regional response indicated universal availability. A separate survey found that no UK ambulance service has access to air cylinders, compressors or ultrasonic nebulisers for COPD patients so all nebulised treatment is oxygendriven. Six of 15 services reported that they had protocols to limit the duration of oxygen-driven nebuliser therapy for COPD patients. Two services have a record of all patients in their area who have an Oxygen Alert card. This survey suggests that UK Ambulance Services are taking steps to implement the BTS and JRCALC emergency oxygen guidance but it is unclear how much advice and equipment had been cascaded to front-line staff by Spring 2011.

\section{P102 A SURVEY OF HOME OXYGEN PROVISION ACROSS LONDON}

doi:10.1136/thoraxjnl-2011-201054c.102

A C Davidson, S Williams, N Baxter, E Morris, L Restrick. London Respiratory Team, NHS London, Victoria, London, UK

Introduction In preparing for 2012, we undertook a survey of current oxygen provision across London.

Methods A questionnaire was sent electronically (Survey Monkey) to 100 BTS Oxygen champions, oxygen leads in primary and secondary care, PCT prescribing advisors and community respiratory nurses around London.

Results From 60 returns the main findings were:

1. $75 \%$ of LTOT prescriptions initiated by hospitals yet no follow-up in $35 \% .70 \%$ community teams reported "not at all" and "only sometimes" being informed about new starters 